\title{
LIMITING POSITIONS SYNTHESIS OF SEVEN BAR SLIDER AUTOMATED FIBER PLACEMENT MECHANISM FOR MOTION GENERATION USING VARIABLE TOPOLOGY
}

\author{
PRASHANT TADALAGI $^{1} \&$ SHRINIVAS BALLI ${ }^{2}$ \\ ${ }^{1}$ Research Scholar, Department of Mechanical Engineering, Basaveshwar Engineering College (Autonomous), Bagalkot, \\ Karnataka, India \\ ${ }^{2}$ Professor and Head, Department of Mechanical Engineering, Basaveshwar Engineering College (Autonomous), Bagalkot, \\ Karnataka, India
}

\begin{abstract}
To produce the high quality production and in mass production, industrialists are more keen to develop mechanisms to perform with greater speed and with high accuracy. The mechanism with multi-tasking is key factor to resolve this. Synthesis of these mechanisms is a difficult process, because it uses higher pairs with higher Degrees of Freedom (DOF). There are numerous techniques utilized for the synthesis of mechanisms with higher links and with higher degrees-offreedom which are complex in nature prompting to time-consuming. Variable Topology Mechanism is the analytical technique used to synthesize the higher DOF mechanism with no iteration. The major factor for a researcher to focus on the operation of mechanism is its limiting or toggle positions. Because these limiting positions decide the operational space and the presence of a dead center in a designed mechanism could be an unwanted structure circumstance if a continuous movement is needed. Nevertheless, it has numerous practical applications as locking devices to make sure about movable members. So this paper is focused on the operating of seven bar slider mechanism with two DoF using variable topology mechanism. In this paper, Grashof type I and Grashof type II mechanisms are used to synthesize using complex number approach. These mechanisms are operating between the limiting positions named as dead center position for Grashof I and Extreme position for Grashof II mechanism. Applications in the automated fiber placement (AFP) are also illustrated.
\end{abstract}

KEYWORDS: Complex Number, Dimensional Synthesis, Dead Center \& Extreme Position Synthesis

Received: Jun 08, 2020; Accepted: Jun 28, 2020; Published: Sep 16, 2020; Paper Id.: IJMPERDJUN20201267

\section{INTRODUCTION}

In the current modernized and technological era, people are more relying upon the machines. To fulfill the requirements of present population with high quality, mass production and worldwide supply, it is necessitated to develop a machine to do multiple tasks in less time with high precision and accuracy. The sole of the machine is its mechanisms. But every mechanism has its limitations in dimensional aspects, performance of linkage/s and operational space. Overcoming of these limitations is easy when the machine operates with lower pairs and single degrees of freedom. But only with lower pairs and single DOF, the mechanisms can't perform multiple tasks. This will in general utilize higher pairs and/or a higher DOF system.

Synthesis of the mechanism includes developing and determining the link lengths and their orientations to play out the necessary task. There are numerous techniques utilized for the synthesis of mechanisms with higher links and with higher degrees-of-freedom which are complex in nature prompting tedious and time-consuming. But, 
these mechanisms can be modified into variable topology mechanism which operates in multiple phases depending on the number of degrees of freedom of original mechanism.

Limiting positions are the major factors to be focused by the researcher to ensure smooth and continuous running of the mechanism. These limiting positions are not drawbacks in all the cases. Sometimes they are converted into useful one also. Limiting positions are named as dead center position in case of Grashof's I and as extreme position in case of Grashof's II type mechanisms [1]. So this paper is focused on the synthesis of seven bar slider with two DOF for both Grashof's types mechanisms operating in their limit/toggle positions.

\section{Variable Topology Mechanism Operation}

Synthesis will be difficult when the mechanism is having higher DOF. So the basic motive for the VTM is to convert these higher DOF mechanisms into single DOF in multiple phases based on the DOF[2], so that the mechanisms could be synthesized easily. To lessen the degrees of freedom, a link is fixed temporarily. This link is next to the permanently fixed link. This results in converting from two DOF to single DOF mechanism. In this phase all link lengths are determined by complex number approach. In next phase, the previously temporarily fixed link is allowed to move and another link is fixed temporarily. This link is likewise ideally is next to the permanently fixed one. This procedure is followed in each phase so that the mechanism reduces to have single DOF in each phase and could be easily synthesized.

Until the 1960s, researchers used graphical methods to synthesize the higher DOF mechanism. Rawat [3] shows synthesizing process of 5 bar mechanism with Variable Topology Method. Joshi and Amaranth [4] built up the VTM by involving Loop Closure equations in it. Balli and Chand examined a technique to synthesize the planar 5-bar mechanism of VTM type for movement between extreme positions[5] which liberates the mechanism from order defect[6], additionally with the transmission angle control[7], they have dealt with the 7-link VTM for two dead-center positions[8]. These papers illuminate an investigative strategy to determine the links of mechanism and potential approaches to reduce the solution space with lesser iterations.

Daivagna and Balli examined the synthesis procedure for seven-bar slider mechanism with VTM for motion between dead-center positions[9]; triad and dyad synthesis of 7 bar mechanism with variable topology for the assignment like function generation [10][11]; In these papers, the authors built up a strategy to synthesize a 5-bar slider with VTM. Dyadic complex number procedure is utilized to compose motion equations and increase the accuracy over graphical procedure and furthermore they determined the FSP Synthesis of an off-set 5 bar-slider with variable topology[12][13].

The review of papers indicates the scope for research to convert the 7-bar slider mechanism into VTM for the applications such as Automated Fiber Placement (AFP). Hence in this paper, the author focused on the synthesis of 7 bar slider mechanism using VTM with Grashof type I \& II operating between limiting/toggle mechanism. With complex number and dyadic approaches, analytical equations are written and solved.

\section{SEVEN BAR SLIDER MECHANISM}

The seven bar slider mechanism of two degrees of freedom is as appeared in figure 1. In which $\mathrm{O}_{a} \mathrm{O}_{b}$ and $\mathrm{O}_{b} \mathrm{O}_{c}$ are permanent links and slider D slides alongside the link $\mathrm{O}_{\mathrm{b}} \mathrm{B}$. This mechanism is of 2 DOF; hence the proposed VTM is operating in two Phases. 


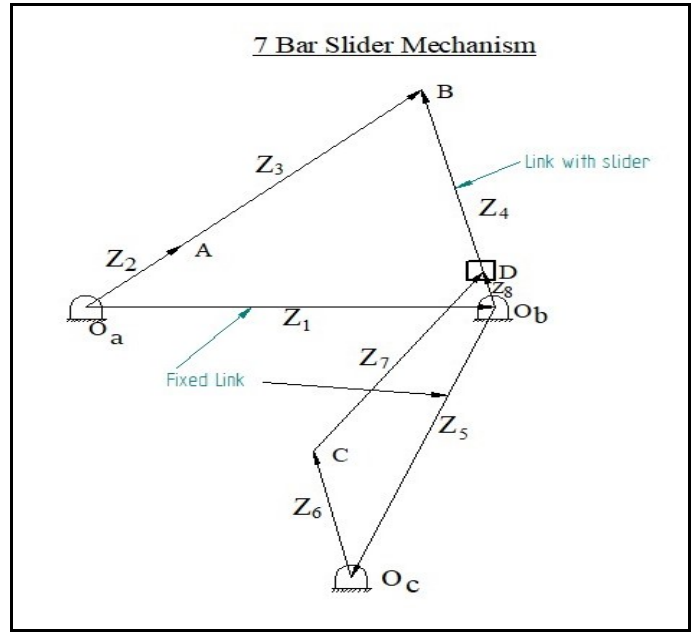

Figure 1: 7 Bar Slider Mechanism.

The conventions followed in the present work is provided in table 1.

Table 1: Conventions used to Indicate the Linkages and The Angles in Both the Phases

\begin{tabular}{|c|c|c|}
\hline Link Vectors and Angular Motion & $\begin{array}{c}\text { Phase - I } \\
\text { From Position 1 to 2 }\end{array}$ & $\begin{array}{c}\text { Phase - II } \\
\text { From Position 2 to 3 }\end{array}$ \\
\hline $\mathrm{O}_{\mathrm{a}} \mathrm{O}_{\mathrm{b}}=\mathrm{Z}_{1}$ & Permanently fixed & Permanently fixed \\
\hline $\mathrm{O}_{\mathrm{b}} \mathrm{O}_{\mathrm{c}}=\mathrm{Z}_{5}$ & Permanently fixed & Permanently fixed \\
\hline $\mathrm{O}_{\mathrm{a}} \mathrm{A}=\mathrm{Z}_{2}, \phi$ & Input Link, $\phi_{12}$ & Temporarily fixed \\
\hline $\mathrm{A}_{1} \mathrm{~B}_{1}=\mathrm{Z}_{3}, \alpha$ & $\alpha_{12}$ & ---- \\
\hline $\mathrm{O}_{\mathrm{b}} \mathrm{B}_{1}=\mathrm{Z}_{4}, \beta$ & $\beta_{12}$ & ---- \\
\hline $\mathrm{O}_{\mathrm{c}} \mathrm{C}_{1}=\mathrm{Z}_{6}, \psi$ & Temporarily fixed & $\Psi_{23}$ \\
\hline $\mathrm{C}_{1} \mathrm{D}_{1}=\mathrm{Z}_{7}, \gamma$ & $\gamma_{12}$ & $\gamma_{23}$ \\
\hline $\mathrm{O}_{\mathrm{b}} \mathrm{D}_{1}=\mathrm{Z}_{8}, \beta$ & $\beta_{12}$ & ---- \\
\hline $\mathrm{O}_{\mathrm{b}} \mathrm{D}_{2}{ }^{\prime}=\mathrm{Z}_{9}, \beta$ & $\beta_{12}$ & --- \\
\hline $\mathrm{O}_{\mathrm{b}} \mathrm{C}=\mathrm{Z}_{10}$ & & \\
\hline $\mathrm{X}_{12}, \mathrm{X}_{23}$ & Slider Displacement & Slider Displacement \\
\hline $\mathrm{B}_{1} \mathrm{~B}_{2}=\mathrm{d}$ & Displacement Vector & ----- \\
\hline Sign Convention & CCW motions are +ve, CW motions are -ve. \\
\hline
\end{tabular}

\section{Dead Centre Position Synthesis of 7 Bar Slider Mechanism}

- A mechanism said to be in dead-center positions, i.e., in locking positions, ought to have zero mechanical advantage [14]. A mechanism is in dead-center positions if, and just if, the input link has more than one instant center with respect to the frame, while the output link has a unique one.

- Based on this theory, the dead center positions are defined for the present mechanism as when the input link and coupler are inline. i.e. when they are in outer in-line position $\left(\Pi\right.$ or $\left.180^{\circ}\right)$ and when overlapped $\left(0^{\circ}\right)$. Hence in this phase, the mechanism will operate from one dead center position (Position 1) to another dead center position (Position 2).

- The proposed mechanism shown in figure 1 is of two DOF. As per methodology of VTM, the synthesis is done in two phases. In each phase the mechanism is converted to single DOF system and determine the link lengths and their orientations. The links determined in Phase-I are retained in Phase-II also. 


\section{Phase-I Synthesis}

In Phase - I synthesis, link $\mathrm{O}_{c} \mathrm{C}$ which is the link next to fixed link $\mathrm{O}_{b} \mathrm{O}_{c}$ is temporarily fixed and input is provided to link $\mathrm{O}_{\mathrm{a}} \mathrm{A}$. After fixing the link; the mechanism will be changed over into 6 bar slider mechanism with single DOF as appeared in Figure 2. $\mathrm{O}_{c} \mathrm{C}$ when temporirly fixed, $\mathrm{O}_{b} \mathrm{O}_{c}$ and $\mathrm{O}_{c} \mathrm{C}$ become one rigid link prompts development of vector $\mathrm{Z}_{10}$. In this phase when the input motion is given to link $\mathrm{O}_{\mathrm{a}} \mathrm{A}$, the mechanism will move from position 1 to 2 .

Now, $\mathrm{O}_{\mathrm{a}} \mathrm{A}_{1}$ is the input link and $\mathrm{A}_{1} \mathrm{~B}_{1}$ is the coupler. The angular motion of the coupler link $\mathrm{A}_{1} \mathrm{~B}_{1}$ to $\mathrm{A}_{2} \mathrm{~B}_{2}$ is $\alpha_{12}$. By definition, the angle between the coupler $\left(\mathrm{A}_{1} \mathrm{~B}_{1}\right)$ and follower link $\left(\mathrm{O}_{\mathrm{b}} \mathrm{B}_{1}\right)$ is known as the Transmission angle and is signified by $\mu[15]$. The slider $\mathrm{D}$ slides from position $\mathrm{D}_{1}$ to $\mathrm{D}_{2}$ along interface link $\mathrm{O}_{\mathrm{b}} \mathrm{B}_{1}$. Since this link is having angular movement, the slider also experience both sliding and angular motion.

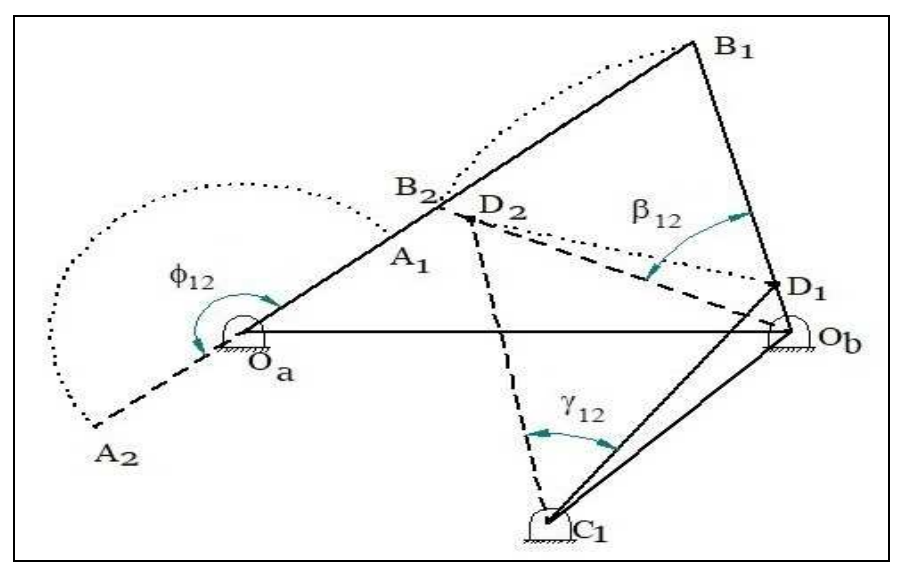

Figure 2: Phase-I Synthesis Mechanism Operating between Dead Center Positions.

The conventions followed in Phase - I are given in Table 1. The input motion in Phase -I is $\phi_{12}$. The displacement vector $B_{1} B_{2}$ is given by $d_{12}$. Referring to Figure 2 , the dyad equations for Phase $-\mathrm{I}$ are as follows:

$$
\begin{aligned}
& Z_{2}\left(e^{i \phi_{12}}-1\right)+Z_{3}\left(e^{i \alpha_{12}}-1\right)=\delta_{12} \\
& Z_{4}\left(e^{i \beta_{12}}-1\right)=\delta_{12} \\
& Z_{9} e^{i \beta_{12}}-Z_{8}=X_{12} \\
& Z_{7}\left(e^{i \gamma_{12}}-1\right)=X_{12}
\end{aligned}
$$

Vector loop equations are

$$
\begin{aligned}
& Z_{1}=Z_{2}+Z_{3}-Z_{4} \\
& Z_{10}=Z_{8}-Z_{7}
\end{aligned}
$$

In the standard dyad Eqns. (1) to (4), for motion generation problem, the coupler point motions $\left(\alpha_{12}, \gamma_{12}\right)$ and displacement vector $d_{12}$ are specified[16]. For 2 position synthesis and from dyad equations, the number of free choices is determined as the difference between the number of unknowns and the number of scalar equations. Here, in this case, the number of unknowns is 16 , and the number of scalar equations is 8 , hence the number of free choices is $16-8=8$. Hence, there will be eight free choices leading to the number of solutions as $\infty^{8}$. 
From dead center definition, the input angle $\phi_{12}$ is equal to $180^{\circ}$. Let us consider the condition that the displacement vector $d_{12}$ and input vector $Z_{2}$ are inline. From figure 2, the coupler motion angle $\alpha_{12}$ is zero.

i.e. $\phi_{12}=180^{\circ}$ and $\alpha_{12}=0^{\circ}$.

From equation (1) by considering value $Z_{3}$ as a free choice and substituting above values, we can find the value of $\mathrm{Z}_{2}$.

As per the description of the problem, position $1 \& 2$ are at dead center positions as shown in Figure 2. From Triangle $\mathrm{O}_{b} \mathrm{~B}_{1} \mathrm{~B}_{2}$

$$
\mathrm{O}_{\mathrm{b}} \mathrm{B}_{2} \mathrm{~B}_{1}=\alpha_{12}
$$

The transmission angle is minimum at position 1. i.e. by the definition of transmission angle and by referring the Figure 1, transmission angle $\mathrm{O}_{b} \mathrm{~B}_{1} \mathrm{~B}_{2}=\mu_{\text {min }}$. As $\mathrm{O}_{b} \mathrm{~B}_{1}$ and $\mathrm{O}_{b} \mathrm{~B}_{2}$ are equal, $\mathrm{O}_{b} \mathrm{~B}_{1} \mathrm{~B}_{2}$ forms an isosceles triangle (References....). So

$$
\begin{aligned}
& \angle \mathrm{O}_{\mathrm{b}} \mathrm{B}_{1} \mathrm{~B}_{2}=\mathrm{b}_{\mathrm{b}} \mathrm{B}_{2} \mathrm{~B}_{1}=\alpha_{12}=\mu_{\text {min }} . \\
& \beta_{12}=180^{\circ}-2 \mu_{\min }
\end{aligned}
$$

The vectors $\mathrm{Z}_{4}, \mathrm{Z}_{8}$, and $\mathrm{Z}_{9}$ are inline, Then by the vector algebra,

Angle of Vector $Z_{4}=$ Angle of Vector $Z_{8}=$ Angle of Vector $Z_{9}[17]$

$$
\text { So, } \tan ^{-1}\left(\frac{Z_{4 y}}{Z_{4 x}}\right)=\tan ^{-1}\left(\frac{Z_{8 y}}{Z_{8 x}}\right)=\tan ^{-1}\left(\frac{Z_{9 y}}{Z_{9 x}}\right)
$$

Since the orientation of $Z_{4}$ is found by the equation (2), if anyone coordinator of $Z_{8}$ and $Z_{9}$ vector is assumed, then remaining coordinate would found out by the above equations.

Hence, from the above equations, the 4 parameters are prescribed and hence the solution space will reduce from $\infty^{8}$ to $\infty^{4}$. Now $Z_{3}, Z_{8}$, and $Z_{9}$ are free choices and $Z_{2}, Z_{4}, X_{12}, Z_{1}, Z_{10}$, and $Z_{7}$ are to be found out by the equations (1) to (7).

\section{Phase-II Synthesis}

In phase - II, the previously short timely fixed link $\mathrm{CO}_{c}$ will be released and link $\mathrm{O}_{\mathrm{a}} \mathrm{A}$ is going to be fixed temporarily. The input motion is provided to link $\mathrm{O}_{c} \mathrm{C}$. Now, this mechanism will act as a slider mechanism as shown in Figure 3. When the input motion is given, the slider will move from $\mathrm{D}_{2}$ to $\mathrm{D}_{3}$. Once the link $\mathrm{O}_{a} \mathrm{~A}_{2}$ is fixed temporarily, the portion $\mathrm{O}_{a} \mathrm{~A}_{2} \mathrm{~B}_{2} \mathrm{O}_{b} \mathrm{O}_{a}$ is resulted in 3 bar truss element. Hence there is no motion for links $\mathrm{O}_{a} \mathrm{~A}_{2}, \mathrm{~A}_{2} \mathrm{~B}_{2}$ and $\mathrm{O}_{b} \mathrm{~B}_{2}$. Also the slider will have only a linear motion. 


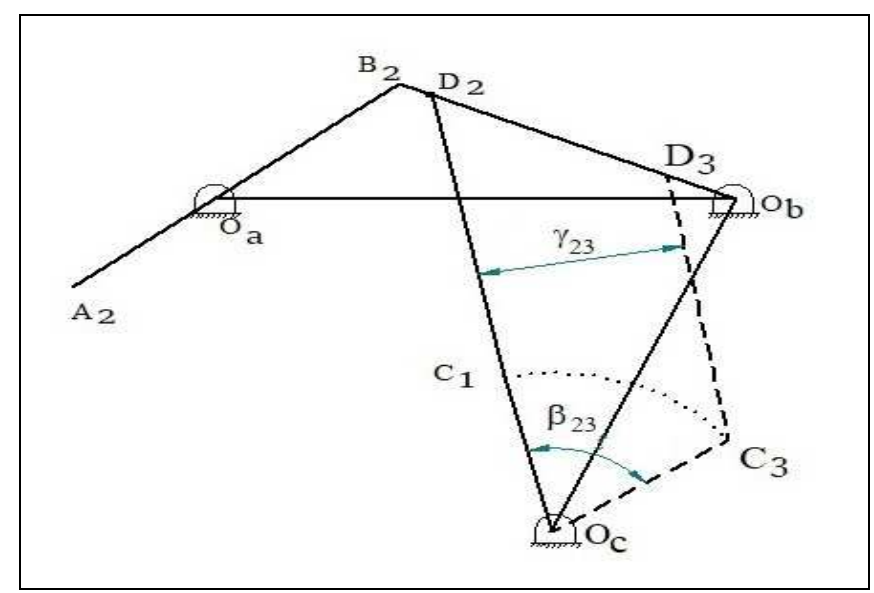

Figure 3: Phase-II Synthesis Mechanism.

The conventions followed in Phase - II are provided in Table 1. The input motion in Phase -II is $\psi_{23}$. The slider displacement is given by $\mathrm{X}_{23}$.

Writing the loop closure equation with reference to Figure 3.

$$
Z_{5}+Z_{6}+Z_{7} e^{i \gamma_{12}}-Z_{9} e^{i \beta_{12}}=0
$$

After giving the input rotation of $\psi_{23}$,

$$
Z_{5}+Z_{6} e^{i \psi_{23}}+Z_{7} e^{i \gamma_{12+23}}-Z_{9} e^{i \beta_{12}} \rho_{23}=0
$$

Where $\rho_{23}=\frac{Z_{9} e^{i \beta_{12}}+X_{23}}{Z_{9} e^{i \beta_{12}}}$ is the stretch ratio.

Subtracting the equation (8) from (9)

$$
\begin{gathered}
Z_{6}\left(e^{i \psi_{23}}-1\right)+Z_{7} e^{i \gamma_{12}}\left(e^{i \gamma_{23}}-1\right)-Z_{9} e^{i \beta_{12}}\left(\rho_{23}-1\right)=0 \\
Z_{10}=Z_{5}+Z_{6}
\end{gathered}
$$

The coupler motion parameters $\gamma_{23}$ and $X_{23}$ are prescribed and $Z_{7} e^{i \gamma_{12}}, Z_{9} e^{i \beta_{12}}$, and $Z_{10}$ are retained from Phase-I. Here, in this case, the number of unknowns is 3 and the number of scalar equations is 2 , hence number of free choices will be $(3-2=1)$. The solution space will be $\infty^{1} . \Psi_{23}$ will be prescribed and the value of $Z_{6}$ and $Z_{5}$ are found out by the equations (11) \& (12).

\section{Extreme Position Synthesis of 7 Bar Slider Mechanism}

In the present work, the two positions considered in Phase-I are at the extreme positions. i.e. when the input link and coupler are along one line at one extreme position and follower and coupler are inline at another extreme position (Reference...). So the transmission angle is at some value in one Phase-I and reaches $180^{\circ}$ in Phase-II. When the transmission angle is $180^{\circ}$, the mechanism will be self-locked which is desirable for operating in Phase-II. 


\section{Phase-I Synthesis}

In Phase - I synthesis, link $\mathrm{O}_{c} \mathrm{C}$ which is the link next to fixed link $\mathrm{O}_{b} \mathrm{O}_{c}$ which is temporarily fixed and input is given to link $\mathrm{O}_{\mathrm{a}} \mathrm{A}$. After fixing the link; the mechanism will be converted into 6 bar slider mechanism with single DOF as shown in Figure 4. $\mathrm{O}_{c} \mathrm{C}$ when temporirly fixed, $\mathrm{O}_{b} \mathrm{O}_{c}$ and $\mathrm{O}_{c} \mathrm{C}$ become one rigid link leading to formation of vector $\mathrm{Z}_{10}$. In this phase when the input motion is given to link $\mathrm{O}_{\mathrm{a}} \mathrm{A}$, the mechanism will move from position 1 to 2 .

Now, $\mathrm{O}_{a} \mathrm{~A}_{1}$ is the input link and $\mathrm{A}_{1} \mathrm{~B}_{1}$ is the coupler. The angular motion of the coupler link $\mathrm{A}_{1} \mathrm{~B}_{1}$ to $\mathrm{A}_{2} \mathrm{~B}_{2}$ is $\alpha_{12}$. By definition, the angle between the coupler $\left(\mathrm{A}_{1} \mathrm{~B}_{1}\right)$ and follower link $\left(\mathrm{O}_{b} \mathrm{~B}_{1}\right)$ is known as the Transmission angle and is denoted by $\mu[15]$. The slider $D$ will slide from position $D_{1}$ to $D_{2}$ along link $O_{b} B_{1}$. Because this link is having angular motion, the slider also experience both sliding and angular motion.

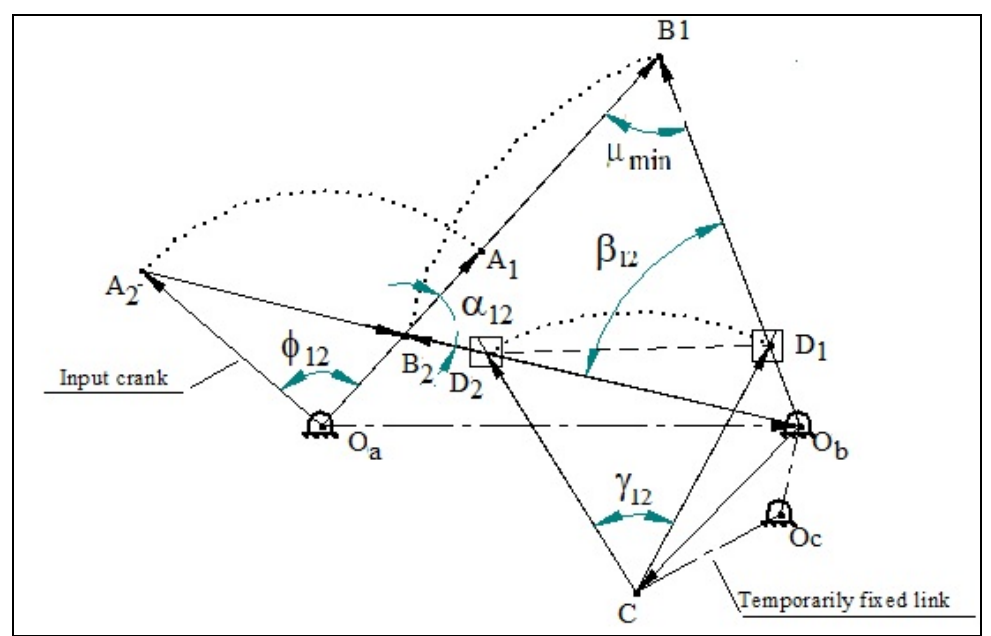

Figure 4: Phase-I Synthesis Mechanism Operating between Extreme Positions.

The conventions followed in Phase - I are given in Table 1. The input motion in Phase - $\mathrm{I}$ is $\phi_{12}$. The displacement vector $B_{1} B_{2}$ is given by $d_{12}$. The dyad and vector loop equations from (1) to (6); used in dead center position are applied in this case also. In the standard dyad Eqns. (1) to (4),

For motion generation problem, the coupler point motions $\left(\alpha_{12}, \gamma_{12}\right)$ and displacement vector $d_{12}$ are specified. Based on the equation of motion and prescribed parameters, the number of free choices is 8 . Hence the solution space is also $\infty^{8}$.

Let us consider the condition that the displacement vector $d_{12}$ and input vector $Z_{2}$ are inline. Then by the vector algebra,

The angle of vector $d_{12}=$ Angle of Vector $Z_{2}$

$$
\text { i.e. } \tan ^{-1}\left(\frac{\delta_{12 Y}}{\delta_{12 x}}\right)=\tan ^{-1}\left(\frac{Z_{2 y}}{Z_{2 x}}\right)
$$

Hence $d_{12}$ is prescribed and one coordinate of $Z_{2}$ is assumed, remaining coordinate can be determined. Similarly, vector $Z_{4}, Z_{8}$, and $Z_{9}$ are inline. As per the description of the problem, the equation (7) is valid here also. And

$$
\beta_{12}=180^{\circ}-2 \mu_{\min }
$$


Now $Z_{2}, \phi_{12}, Z_{8}$, and $Z_{9}$ are free choices and $Z_{3}, Z_{4}, X_{12}, Z_{1}, Z_{10}$, and $Z_{7}$ are to be found out by the equations (1) to (9). Hence the solution space is reduced from $\infty^{8}$ to $\infty^{4}$.

\section{Phase-II Synthesis}

In phase - II, the previously short timely fixed link $\mathrm{CO}_{\mathrm{c}}$ will be released and link $\mathrm{O}_{\mathrm{a}} \mathrm{A}$ is going to be fixed temporarily. The input motion is provided to link $\mathrm{O}_{c} \mathrm{C}$. Now, this mechanism will act as a slider mechanism as shown in Figure 3 . When the input motion is given, the slider will move from $D_{2}$ to $D_{3}$. Once the link $\mathrm{O}_{a} A_{2}$ is fixed temporarily, the portion $\mathrm{O}_{a} A_{2} B_{2} O_{b} O_{a}$ is resulted in 3 bar truss element. Hence there is no motion for links $\mathrm{O}_{\mathrm{a}} \mathrm{A}_{2}, \mathrm{~A}_{2} \mathrm{~B}_{2}$ and $\mathrm{O}_{\mathrm{b}} \mathrm{B}_{2}$. Also the slider will have only a linear motion.

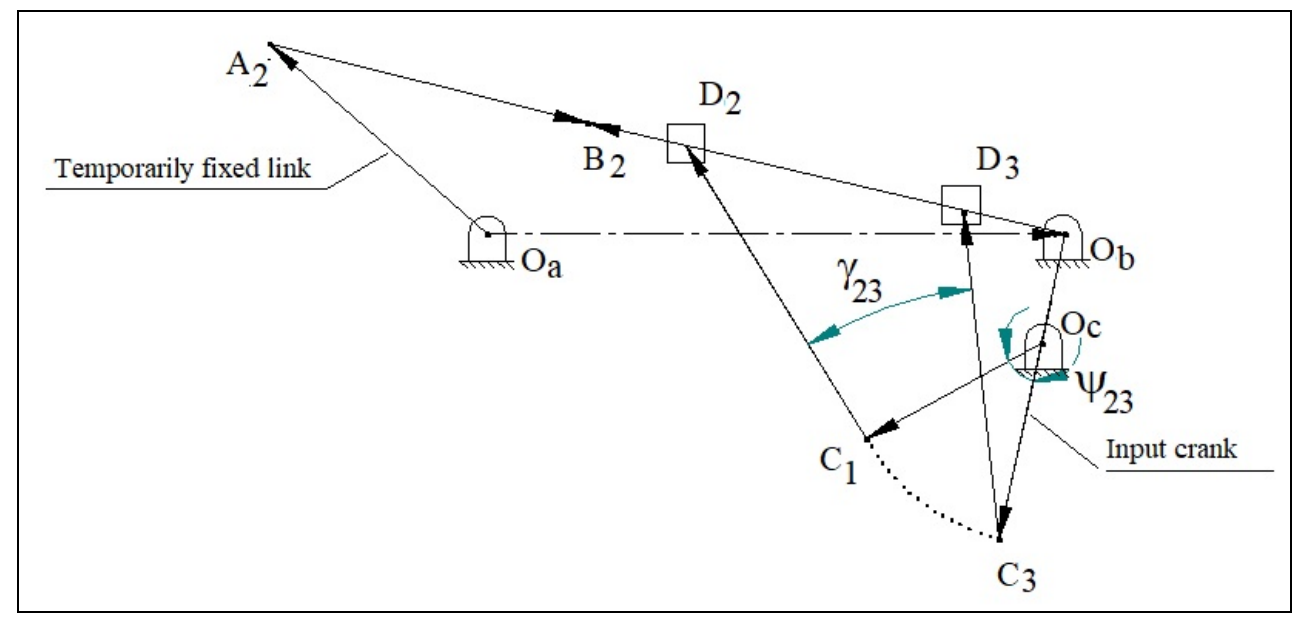

Figure 5: 7 Bar Slider Variable Topology Mechanism in Phase-II.

The conventions followed in Phase - II are provided in Table 1. The input motion in Phase -II is $\psi_{23}$. The slider displacement is given by $\mathrm{X}_{23}$. The dyad and vector loop equations from (8) to (12) is applicable here also.

The coupler motion parameters $\gamma_{23}$ and $\mathrm{X}_{23}$ are prescribed and $\mathrm{Z}_{7} \mathrm{e}^{\mathrm{i} \gamma_{12}}, \mathrm{Z}_{9} \mathrm{e}^{\mathrm{i} \beta_{12}}$, and $\mathrm{Z}_{10}$ are retained from Phase-I. Here, in this case, the number of unknowns is 3 and the number of scalar equations is 2 , hence number of free choices will be $(3-2=1)$. The solution space will be $\infty^{1} . \Psi_{23}$ will be prescribed and the value of $Z_{6}$ and $Z_{5}$ are found out by the equations (11) \& (12).

\section{CONCLUSIONS}

The proposed technique presented in this paper is for the seven link slider mechanism with two DOF. In this context, mechanism operates between its limiting positions for both Grashof type I \& II mechanism. This mechanism operates in two phases, in which the higher DOF mechanism is reduced to a single DOF mechanism in each phase. The method is simple and the single mechanism can perform multiple tasks. This method reduces the solution space with no iteration.

\section{EXAMPLES}

The presented mechanism is illustrated by the following examples.

Example 1: It is required to synthesize the planar 7-bar mechanism acting between its dead center positions (Grashof's type I) as in Figure 1. Provided that coupler parameters $d_{12}=-45.882-i \times 38.663, \alpha_{12}=0^{\circ} \mathrm{CCW} . \gamma_{12}=50^{\circ}$ CCW. 

Motion Generation using Variable Topology

Solution: Consider the mechanism to operate in two phases as shown in Figure $2 \& 3$.

In Phase-I, the mechanism operates between dead center positions. The transmission angle is in the range of $60^{\circ} \leq$ $\mu \leq 180^{\circ}$.

Let $\mu_{\min }=61^{\circ}$, From Figure $2, \beta_{12}=180^{\circ}-2 \times \mu_{\min }=180-2 \times 61=58^{\circ} \mathrm{CCW}$

Free choices made: $Z_{3}=49.706+i \times 41.885$,

The real part of vectors $Z_{8} \& Z_{9} . \quad Z_{8 X}=-1.978, \quad Z_{9 X}=-10.814$

From equations (1) to (7) and upon substituting these above values, the vectors $Z_{2}, Z_{4}, X_{12}, Z_{7}, Z_{1}$ and $Z_{10}$ are determined.

Phase - II, Given $\mathrm{X}_{23}=37.323-i \times 14.446, \gamma_{23}=1^{\circ} \mathrm{CW}$. and retaining the values of vectors, $\mathrm{Z}_{9} \mathrm{e}^{\mathrm{i} \beta_{12}}$, $\mathrm{Z}_{7} \mathrm{e}^{\mathrm{i} \gamma_{12}}$ from Phase-I. The free choice made is $\psi_{23}=69^{\circ} \mathrm{CW}$. The value of vectors $\mathrm{Z}_{6}$ and $\mathrm{Z}_{5}$ are determined from (10) to (12) and tabulated in below table 2.

Table 2: Length and Orientation of all Links of the Mechanism for Dead Center Position Synthesis

\begin{tabular}{|c|c|c|c|c|}
\hline Sl. No. & Vector & Position & Magnitude & $\begin{array}{c}\text { Angle measured from } \\
\text { +ve X-axis CCW }\end{array}$ \\
\hline 1 & $\mathrm{Z}_{1}$ & $\mathbf{8 5}$ & 85 & $0^{\circ}$ \\
\hline 2 & $\mathrm{Z}_{2}$ & $22.9410+i \times 19.3310$ & 30 & $40.11^{\circ}$ \\
\hline 3 & $\mathrm{Z}_{3}$ & $49.706+i \times 41.835$ & 65 & $40.11^{\circ}$ \\
\hline 4 & $\mathrm{Z}_{4}$ & $-12.353+i \times 61.2160$ & 64.44 & $101.41^{\circ}$ \\
\hline 5 & $\mathrm{Z}_{5}$ & $-\mathbf{- 2 9 . 3 1 1 - i \times 7 3 . 3 4 6}$ & 79 & $248.21^{\circ}$ \\
\hline 6 & $\mathrm{Z}_{6}$ & $-\mathbf{- 8 . 0 1 2}+i \times 34.692$ & 35.605 & $103^{\circ}$ \\
\hline 7 & $\mathrm{Z}_{7}$ & $\mathbf{3 5 . 3 4 6 + i \times 4 8 . 4 5 6}$ & 60 & $53.89^{\circ}$ \\
\hline 8 & $\mathrm{Z}_{8}$ & $-\mathbf{- 1 . 9 7 8 + i \times 9 . 8 0 2}$ & 10.00 & $101.41^{\circ}$ \\
\hline 9 & $\mathrm{Z}_{9}$ & $-\mathbf{- 1 0 . 8 1 4 + i \times 5 3 . 5 8 9}$ & 54.66 & $101.41^{\circ}$ \\
\hline 10 & $\mathrm{Z}_{10}$ & $-\mathbf{- 3 7 . 3 2 4 - i \times 3 8 . 6 5 4}$ & 53.752 & $226^{\circ}$ \\
\hline
\end{tabular}

Example 2: It is required to synthesize a planar 7-bar mechanism with VTM operating between extreme positions as in Figure 1. Provided that coupler parameters $d_{12}=-32.278-i$ x $36.97, \alpha_{12}=62^{\circ} \mathrm{CW} \cdot \gamma_{12}=60^{\circ} \mathrm{CCW}$.

Solution: Consider the mechanism to operate in two phases as shown in Figure $4 \&$ 5. In Phase-I, the mechanism operates between extreme positions. The transmission angle is in the range of $60^{\circ} \leq \mu \leq 180^{\circ}$.

Let $\mu_{\min }=62^{\circ}$, From Figure $3, \beta_{12}=180^{\circ}-2 \times \mu_{\min }=180-2 \times 62=56^{\circ}$

Let be consider the condition that the displacement vector $d_{12}$ and input vector $Z_{2}$ are inline as shown in figure 4 .

Free choices made: $Z_{2 x}=19.702, \phi_{12}=91^{\circ}, Z_{8 x}=-3,5, Z_{9 x}=-13.956$

From equations (1) to (7) and equation (13), the values of vectors $\mathrm{Z}_{3}, \mathrm{Z}_{4}, \mathrm{X}_{12}, \mathrm{Z}_{7}, \mathrm{Z}_{1}$ and $\mathrm{Z}_{10}$ are determined.

Phase - II

Given $\mathrm{X}_{23}=29.27-\mathrm{i} \times 6.579, \gamma_{23}=25^{\circ} \mathrm{CW}$ and retaining the values of vectors $\mathrm{Z}_{9} \mathrm{e}^{\mathrm{i} \beta_{12}}, \mathrm{Z}_{7} \mathrm{e}^{\mathrm{i} \gamma_{12}}$ from Phase-I. The free choice made is $\psi_{23}=50^{\circ} \mathrm{CCW}$. The value of vectors $Z_{6}$ and $Z_{5}$ are determined from (10) to (12) and tabulated in table 3. 
Table 3: Length and Orientation of all Links of the Mechanism for Extreme Position Synthesis

\begin{tabular}{|c|c|c|c|c|}
\hline Sl. No. & Vector & Position & Magnitude & $\begin{array}{l}\text { Angle measured by } \\
\text { + ve } \mathrm{X} \text {-axis CCW }\end{array}$ \\
\hline 1 & $\mathrm{Z}_{1}$ & 61.127 & 61.127 & $0^{\circ}$ \\
\hline 2 & $\mathrm{Z}_{2}$ & $19.702+i x 22.565$ & 29.98 & $48.87^{\circ}$ \\
\hline 3 & $\mathrm{Z}_{3}$ & $23.513+w 25.888$ & 34.976 & $48.75^{\circ}$ \\
\hline 4 & $\mathrm{Z}_{4}$ & $-17.906+i x 48.209$ & 51.427 & $110.38^{\circ}$ \\
\hline 5 & $\mathrm{Z}_{5}$ & $-1,116-i \times 10,0147$ & 11.742 & $264.54^{\circ}$ \\
\hline 6 & $\mathrm{Z}_{6}$ & $-19.622-i \times 10.0147$ & 22.029 & $207.03^{\circ}$ \\
\hline 7 & $\mathrm{Z}_{7}$ & $17.197+i x 31.107$ & 35.544 & $61.065^{\circ}$ \\
\hline 8 & $\mathrm{Z}_{8}$ & $-3.5+x 9.372$ & 10.00 & $110.38^{\circ}$ \\
\hline 9 & $\mathrm{Z}_{9}$ & $-13.956+i \times 37.486$ & 40 & $110.38^{\circ}$ \\
\hline 10 & $\mathrm{Z}_{10}$ & $-20,697-i x 21,735$ & 30.012 & $225.97^{\circ}$ \\
\hline
\end{tabular}

\section{APPLICATION OF VTM IN AUTOMATED FIBER PLACEMENT MECHANISM}

The underneath model portrays the application of proposed method in Automated Fiber Placement (AFP). In this procedure, the number of resin-impregnated fibers is fed into the fiber placement head dependent on the mould surface. These fibers are pulled from the creel and fed to the CCR unit. In the CCR unit, the fibers are going to cut at the specific size and then these are laid onto the mandrel with radiation and compacted to the mold surface by compacting process as shown in Figure. 6. By interfacing knife blade and clamping block, cutting and clamping activities are handled.

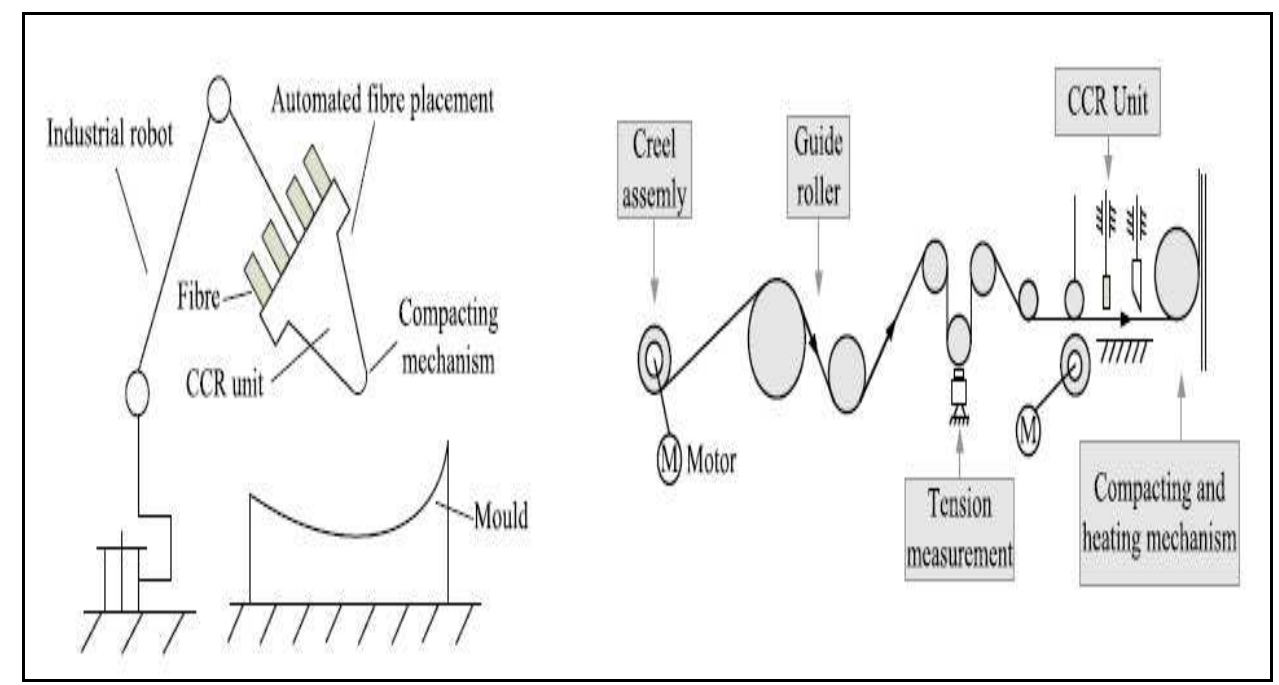

Figure 6: Schematic Diagram of Automated Fiber Placement Machine.[18].

A variable topology mechanism is acquainted to perform the cutting, clamping, and restarting activities as appeared in Figure.7. $\mathrm{O}_{a} \mathrm{O}_{b}$ and $\mathrm{O}_{b} \mathrm{O}_{c}$ are permanent links and $\mathrm{L}$ shaped link BE which is pivoted at $\mathrm{O}_{\mathrm{b}}$ is utilized to engage and disengage the tow. As shown in Figure 2 in Phase - I, the slider D will slide along the link $\mathrm{O}_{\mathrm{b}} \mathrm{B}$ and brings the tow into the mould surface. In Phase - II, as appeared in Figure 3, the slider will move from position $\mathrm{D}_{2}$ to $\mathrm{D}_{3}$ now to toes are chopped and to lay down onto mould with compacting and cutting edge associated with it. This process will lead to automatic fabrication of composites through fibre placement method. 


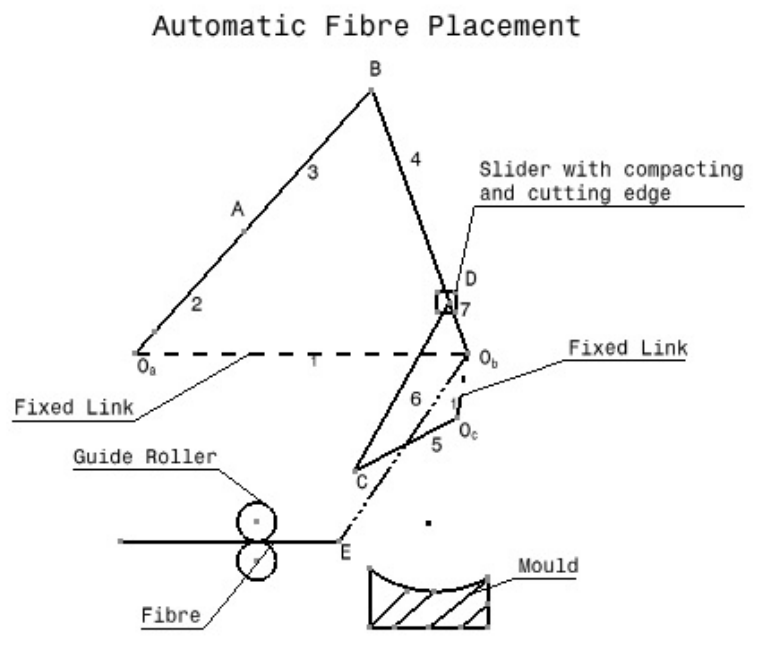

Figure 7: Proposed 7 Bar Slider Variable Topology Mechanism.

\section{REFERENCES}

1. A. K. Khare and R. K. Dave, “Optimizing 4-Bar Crank-Rocker Mechanism,” Mech. Mach. Theory, vol. 14, pp. 319-325, 1979.

2. M. M. Prashant and S. S. Balli, “The Study Of Five-Bar Mechanism With Variable Topology," vol. 102, 2006.

3. Y. R. Rawat, “Synthesis of Variable Topology Mechanism,” IIT Bombay, 1996.

4. Y. R. Rawat. S. A. Joshi, C. Amarnath, "Synthesis of variable Topology Mechanisms for circuit breaker applications," in National Conference on Mechanism and Machines (NaCoMM), 1997.

5. S. S. Balli and S. Chand, "Synthesis of a Five-bar mechanism with variable topology for motion between extreme positions ( SYNFBVTM )," Mech. Mach. Theory, vol. 36, pp. 1147-1156, 2001.

6. S. S. Balli and S. Chand, "Defects in link mechanisms and solution rectification," Mech. Mach. Theory, vol. 37, no. 9, pp. 851-876, 2002.

7. S. S. Balli and S. Chand, "Synthesis of a Five-Bar Mechanism of Variable Topology Type With Transmission Angle," Mech. Des., vol. 126, no. January 2004, pp. 2-8, 2003.

8. S. S. Balli and S. Chand, "Synthesis of a planar seven-link mechanism with variable topology for motion between two deadcenter positions,” Mech. Mach. Theory, vol. 38, no. 11, pp. 1271-1287, 2003.

9. U. M. Daivagna and S. S. Balli, "Synthesis of a seven-bar slider mechanism with variable topology for motion between two dead-center positions," WCE 2010 - World Congr. Eng. 2010, vol. 2, pp. 1454-1459, 2010.

10. S. S. Balli, Gadad. Gururaj M, HV Ramakrishna, M S Srinath, "Dyad synthesis of planar seven-link variable topology mechanism for motion between two dead-centre positions," IOSR J. Mech. Civ. Eng., vol. 3, no. 3, pp. 21-29, 2012.

11. U. M. Daivagna. G. M. Gadad, Shrinivas. S. Balli, "Triad and Dyad Synthesis of Planar Seven-Link Mechanisms with Variable Topology," in National Conference on Mechanism and Machines (NaCoMM), 2005, pp. 67-73.

12. U. M. Daivagna and S. S. Balli, "FSP Synthesis of an off-set five bar-slider mechanism with variable topology 2 Variable Topology of Five-Bar Slider Mechanism,” Natl. Conf. Mech. Mach., pp. 345-350, 2007.

13. U. M. Daivagna and S. S. Balli, "Synthesis of five-bar slider mechanism with variable topology for finitely separated positions," Adv. Mech. Eng., vol. 2011, 2011. 
14. H. Yan and L. Wu, "On the Dead-Center Positions of Planar Linkage Mechanisms," vol. 111, no. March 1989, 2017.

15. C. R. Barker and G. Shu, "Three-Position Function Generation of Planar Four-Bar Mechanisms With Equal Deviation Transmission Angle Control,” Mech. Transm. Autom. Des., vol. 110, no. December 1988, pp. 435-439, 2017.

16. G. N. S. Arthur G Erdman, Mechanism Design: Analysis and Synthesis, Volume 1, 4th ed. New Jersey: Prentice Hall, 1994.

17. https://www.dummies.com/education/science/physics/how-to-find-a-vectors-magnitude-and-direction.

18. W. Zhang, F. Liu, Y. Lv, and X. Ding, "Design and analysis of a metamorphic mechanism for automated fibre placement," Mech. Mach. Theory, vol. 130, pp. 463-476, 2018. 